\title{
REGULARITY OF WEAK SOLUTIONS OF PARABOLIC VARIATIONAL INEQUALITIES
}

\author{
WILLIAM P. ZIEMER
}

ABSTRACT. In this paper, parabolic operators of the form

$$
u_{t}-\operatorname{div} A(x, t, u, D u)-B(x, t, u, D u)
$$

are considered where $A$ and $B$ are Borel measurable and subject to linear growth conditions. Let $\psi: \Omega \rightarrow R^{1}$ be a Borel function bounded above (an obstacle) where $\Omega \subset R^{n+1}$. Let $u \in W^{1,2}(\Omega)$ be a weak solution of the variational inequality in the following sense: assume that $u \geq \psi$ q.e. and

$$
\int_{\Omega} u_{t} \varphi+A \cdot D \varphi-B \varphi \geq 0
$$

whenever $\varphi \in W_{0}^{1,2}(\Omega)$ and $\varphi \geq u-\psi$ q.e. Here q.e. means everywhere except for a set of classical parabolic capacity. It is shown that $u$ is continuous even though the obstacle may be discontinuous. A mild condition on $\psi$ which can be expressed in terms of the fine topology is sufficient to ensure the continuity of $u$. A modulus of continuity is obtained for $u$ in terms of the data given for $\psi$.

1. Introduction. In this paper we consider the question of pointwise regularity of weak solutions of parabolic obstacle problems for parabolic operators of the following type:

$$
u_{t}-\operatorname{div} A(x, t, u, \nabla u)-B(x, t, u, \nabla u),
$$

whose structure is defined by the inequalities

$$
\begin{aligned}
|A(x, t, u, \xi)| & \leq a_{0}|\xi|+a_{1}|u|+a_{2}, \\
\xi \cdot A(x, t, u, \xi) & \geq|\xi|^{2}-c_{1}|u|^{2}-c_{2}, \\
|B(x, t, u, \xi)| & \leq b_{0}|\xi|+b_{1}|u|+b_{2},
\end{aligned}
$$

where $a_{0}$ is a positive constant and the remaining coefficients are nonnegative measurable functions defined on an open bounded set $\Omega \subset R^{n+1}$. For brevity and simplicity of exposition, we will assume that these coefficients are bounded although our results remain valid if they are assumed members of appropriate $L^{p}$-spaces. Our results described below would remain valid if the inequality involving $B$ would include a term of the form $b|\xi|^{2}$, where $b$ is a nonnegative constant, if we would assume that the solution is bounded. The reader may consult [LSU, Chapter V] for details.

The obstacle that we consider is an arbitrary Borel function $\psi$ defined on $\Omega$ that is bounded above. Our main objective is to specify a condition on $\psi$ at a

Received by the editors August 10, 1987.

1980 Mathematics Subject Classification (1985 Revision). Primary 49A29; Secondary 35K10.

Research supported in part by a grant from the National Science Foundation. 
point $z_{0}=\left(x_{0}, t_{0}\right)$ that will ensure the continuity of the weak solution $u$ at $z_{0}$. Stated briefly, we show that $u$ is continuous even though the obstacle $\psi$ may be discontinuous. Moreover, the modulus of continuity of $u$ is estimated in large part by the fine properties (in the sense of parabolic potential theory) of the obstacle. See Theorems 3.7, 4.4, and 4.5.

There is a vast literature on the regularity of solutions to elliptic and parabolic variational inequalities. See [BI, BM, CK, DV, FM, MZ, and SV] for a small selection of papers that are relevant to our situation. Before we discuss the definition of a weak solution to the parabolic obstacle problem, it may be helpful to review the setting of the corresponding elliptic problem that was treated in $[\mathbf{M Z}]$. For this purpose, let $U$ be a bounded nonempty open set of $R^{n}$. Let $K$ be the subset of the Sobolev space $W^{1, p}(U), p>1$, consisting of all $v$, such that $v$ agrees with a boundary function $\theta$ on $\partial U$ in a suitable way and $v(x) \geq \psi(x)$, for almost all $x \in U$, where $\psi$ is a function defined on $U$ (the "obstacle"). Put

$$
I(v)=\int_{U} F(x, v(x), \nabla v(x)) d x
$$

for $v \in K$, where $F$ is a function with suitable properties. Let

$$
\sigma=\inf _{v \in K} I(v)
$$

and suppose there is a function $u \in K$, such that

$$
I(u)=\sigma .
$$

This gives a general description of the classical obstacle problem where $u$ is the solution to the problem.

It is well known that if $u \in K$ is such that (4) holds and the function $F$ satisfies appropriate conditions, then

$$
\begin{aligned}
& \sum_{i=1}^{n} \int_{U} \frac{\partial F}{\partial \xi_{i}}(x, u(x), \nabla u(x)) \frac{\partial \varphi}{\partial x_{i}}(x) d x \\
& \quad+\int_{U} \frac{\partial F}{\partial z}(x, u(x), \nabla u(x)) \varphi(x) d x \geq 0,
\end{aligned}
$$

for all $\varphi \in W_{0}^{1, p}(U)$ with

$$
\varphi(x) \geq \psi(x)-u(x)
$$

for almost all $x \in U$. This is a special case of the weak inequality

$$
\sum_{i=1}^{n} \int_{U} A_{i}(x, u(x), \nabla u(x)) \frac{\partial \varphi}{\partial x_{i}}(x) d x+\int_{U} B(x, u(x), \nabla u(x)) \varphi(x) d x \geq 0
$$

for all $\varphi \in W_{0}^{1, p}(U)$ with

$$
\varphi(x) \geq \psi(x)-u(x)
$$

for almost all $x \in U$. This was the inequality that was investigated in [MZ]. Thus, the definition of weak solution that was employed was based on the assumption that $u \in W^{1, p}(U), p>1$,

$$
u(x) \geq \psi(x)
$$


for almost all $x \in U$ and $u$ satisfied inequality (7) for all $\varphi$ satisfying (8). Our definition of weak solution to the parabolic obstacle problem will be patterned after this one.

The main result in $[\mathbf{M Z}]$ is the following: assume that the obstacle function $\psi$ is a Borel function on $U$ that is bounded above. Let $x_{0} \in U$ and suppose there is a set $A \subset R^{n}$ with the property that $A$ is not thin at $x_{0}$ and that

$$
\lim _{\substack{x \rightarrow x_{0} \\ x \in A}} \psi(x)=\bar{\psi}\left(x_{0}\right),
$$

where $\bar{\psi}\left(x_{0}\right)=\lim _{r \rightarrow 0} \bar{\psi}(r)$ and $\bar{\psi}(r)=\sup \left\{\psi(x): x \in B\left(x_{0}, r\right)\right\}$. Then the solution $u$ is continuous at $x_{0}$. The set $A$ is said to be not thin (thin) at $x_{0}$ if

$$
\int_{0}\left[\frac{\gamma\left[B\left(x_{0}, r\right) \cap A\right]}{\gamma\left[B\left(x_{0}, r\right)\right]}\right]^{1 /(p-1)} \frac{d r}{r}=\infty \quad(<\infty) .
$$

Some authors use the terminology "fat at $x_{0}$ " instead of "not thin at $x_{0}$." Here $p>1$ is a number associated with the structure of the elliptic operator of the obstacle problem and $\gamma$ is a capacity, the so-called $p$-capacity. The $p$-capacity of a set $E$ is defined by

$$
\gamma(E)=\inf \left\{\int_{R^{n}}|\nabla u|^{p}: u \in W^{1, p}\left(R^{n}\right), E \subset \operatorname{int}\{u \geq 1\}\right\} .
$$

This result should be compared to the question of regularity of weak solutions of equations at a point $x_{0}$ of the boundary of an arbitrary open set $U$. It was shown in $[\mathbf{G Z}]$ that a weak solution of an equation of the form (1) is continuous at $x_{0}$ if $R^{n}-U$ is not thin at $x_{0}$. In case the prescribed boundary values are identically one, this problem can be interpreted as an obstacle problem with the characteristic function of $R^{n}-U$ considered as the obstacle. Then condition (10) is equivalent to $R^{n}-U$ being not thin at $x_{0}$.

Proceeding by analogy with the elliptic case, in the parabolic setting we consider the obstacle as a Borel function $\psi$ defined on $\Omega \subset R^{n+1}$ which is assumed to be bounded above. We consider $u \in W^{1,2}(\Omega)$ to be a solution of the parabolic obstacle problem in the following sense: we assume

$$
\int_{\Omega}-\varphi_{t} u+A(x, t, u, \nabla u) \cdot \nabla \varphi-\varphi B(x, t, u, \nabla u) \geq 0
$$

for all $\varphi \in W_{0}^{1,2}(\Omega)$ with

$$
\varphi(z) \geq \psi(z)-u(z)
$$

for almost all $z \in \Omega$. We assume throughout that $u(z) \geq \psi(z)$ for almost all $z \in \Omega$. This definition is analogous to the one employed in the elliptic setting as described in (7) and (8). The analysis of this paper could have been carried through by assuming that $u$ is merely an element of the space $V^{2}(\Omega)$ (see [LSU] for definitions and development). The basic estimates that we obtain can be obtained for these general weak solutions by employing integral averages of the solutions we consider. The notion of thinness in the parabolic context will be expressed in terms of thermal capacity which is defined by

$$
\operatorname{cap}_{T}(K)=\sup \left\{\mu\left(R^{n+1}\right): \mu \in M(K), E * \mu \leq 1\right\}
$$


whenever $K \subset R^{n+1}$ is a compact set. Here $M(K)$ is the set of all nonnegative measures supported by $K, E$ is the fundamental solution of the heat operator $\partial / \partial t-\Delta$, and * denotes the convolution in $R^{n+1}$. With this concept of capacity, we refine the definition of a solution to the parabolic obstacle problem in the following way. We now assume that the obstacle $\psi$ is defined everywhere except for a set of $\operatorname{cap}_{T}$ zero. We will use the expression quasi-everywhere, q.e., to mean everywhere except for a set of $\operatorname{cap}_{T}$ zero. We also assume that

$$
\varphi(z) \geq \psi(z)-u(z) \quad \text { and } \quad u(z) \geq \psi(z)
$$

for q.e. $z \in R^{n+1}$. Finally, we will replace (11) by the expression

$$
\int_{\Omega} u_{t} \varphi+A(x, t, u, \nabla u) \cdot \nabla \varphi-\varphi B(x, t, u, \nabla u) \geq 0
$$

for all $\varphi \geq \psi-u$ q.e., spt $\varphi \subset \Omega$, and $\varphi \in W_{0}^{1,2}\left(\Omega_{t}\right)$ for each $t$. Here,

$$
\Omega_{t}=\Omega \cap\{(x, \tau): \tau=t\} \text {. }
$$

This can be proved by elementary regularization techniques. Observe that a solution of the obstacle problem is automatically a weak supersolution of the equation $u_{t}=\operatorname{div} A+B$.

Regularity of weak solutions of parabolic obstacle problems has been considered by Biroli and Mosco [BM] for operators of the form $u_{t}+L u$. Here $L$ is the linear operator $L(u)=D_{i}\left(a^{i j}(x, t) D_{j} u\right)$ whose coefficients $a^{i j}$ are bounded, measurable functions. The capacity they employ is defined by

$$
\operatorname{cap}(E)=\inf \left\{\int|\nabla v|^{2} d x d t: v \in C_{0}^{\infty}\left(R^{n+1}\right), v \geq 1 \text { on a nhbd of } E\right\} .
$$

Their notion of weak solution of the obstacle problem is as follows: The underlying domain is $Q=U \times[0, T]$. It is assumed that $u \geq \psi$ everywhere except for a set of cap zero, that $u$ is a weak supersolution of the equation $u_{t}+L u=0$, and that for every nonnegative function $\eta \in C_{0}^{\infty}$ and every constant $k$ such that $k \geq \psi$ everywhere except for a set of cap zero, we have

$$
\begin{aligned}
\frac{1}{2} \|(u & -k)^{+} \eta \|_{L^{2}(U)}^{2}(t)-\int_{0}^{t} \int_{U} \eta_{t} \eta\left[(u-k)^{+}\right]^{2} d x d s \\
& +\int_{0}^{t} \int_{U} a^{i j}(x, s) D_{j} u D_{i}\left[(u-k)^{+} \eta^{2}\right] d x d s=0
\end{aligned}
$$

for $0<t<T$. Equivalently, if one defines a test function

$$
\varphi=\eta^{2}(u-k)^{+} \chi_{\{(x, s): 0 \leq s \leq t\}},
$$

where $\chi_{A}$ denotes the characteristic function of a set $A$, then

$$
\int_{0}^{t} \int u_{t} \varphi-\int_{0}^{t} \int_{U} a^{i j}(x, s) D_{j} u D_{i}\left[(u-k)^{+} \eta^{2}\right] d x d s=0 .
$$

They show that their notion of solution is in agreement with those used by Mignot and Puel [MP] and Pierre [P], where the question of existence was investigated. It is shown during the course of the exposition, that the results of this paper still remain valid if this notion of solution had been adopted. 
There were two basic ideas in the paper [MZ] that led to the pointwise regularity of the solution to the obstacle problem. The first was the observation that if $k>\sup \psi$ in some open set $V$, then $(u-k)^{+}$behaves like a subsolution to the equation in $V$. The analogous result is used in the present paper and its proof carries over without difficulty. The second idea was to show that a solution to the obstacle problem (which is a supersolution to the equation) has the property that it is finely continuous at all points in its domain. This was accomplished by obtaining an estimate of the rate of decay of $r^{p-n} \int_{B(x, r)}|\nabla u|^{p}$ as $r \downarrow 0$. This type of analysis is not suitable in the parabolic case and must therefore be replaced by another argument to conclude that the solution of the obstacle problem is finely continuous everywhere. We are able to supply such an argument thus yielding the pointwise regularity of the solution. Our methods employ a combination of the truncation procedure of De Giorgi and the Moser iteration technique.

2. Preliminaries. Points will $R^{n+1}$ will generally be denoted by $z=(x, t)$ where $x=\left(x_{1}, \ldots, x_{n}\right)$. The Sobolev space of real valued functions defined on an open set $\Omega \subset R^{n+1}$ whose distribution first partial derivatives belong to $L^{2}(\Omega)$ $\left(L_{\text {loc }}^{2}(\Omega)\right)$ is denoted by $W^{1,2}(\Omega)\left(W_{\text {loc }}^{1,2}(\Omega)\right)$. The letter $C$ will denote perhaps different constants appearing within a discussion, but with the understanding that they all depend on the same set of parameters. The integral average of a function $u$ will be denoted by $f_{A} u$. Thus, $f_{A} u=|A|^{-1} \int_{A} u$, where $|A|$ denotes the Lebesgue measure of the set $A$. of

A function $u \in W_{\text {loc }}^{1,2}(\Omega)$ is said to be a weak solution (subsolution, supersolution)

in $\Omega$ if

$$
u_{t}=\operatorname{div} A(x, t, u, \nabla u)+B(x, t, u, \nabla u)
$$

$$
\int_{\Omega}-\varphi_{t} u+A(x, t, u, \nabla u) \cdot \nabla \varphi-B(x, t, u, \nabla u) \varphi=0 \quad(\leq 0, \geq 0)
$$

for all nonnegative $\varphi \in W_{0}^{1,2}(\Omega)$.

We now introduce a geometric configuration that will be used throughout the sequel. Consider a point $z_{0}=\left(x_{0}, t_{0}\right) \in \Omega$. For each positive number $r$ let

$$
R(r)=B\left(x_{0}, r\right) \times\left(t_{0}-\frac{3}{4} r^{2}, t_{0}+\frac{1}{4} r^{2}\right)
$$

where $B\left(x_{0}, r\right)$ denotes the open ball in $R^{n}$ centered at $x_{0}$ with radius $r$. Associated with $R(r)$ are the following subcylinders:

$$
\begin{aligned}
& R^{+}(r)=R(r / 2), \\
& D^{+}(r)=R(\tau r / 2) \text { where } \tau \text { is chosen so that }(\tau \cdot r / 2)^{2}<\frac{1}{4} r^{2}, \\
& R^{-}(r) \text { and } D^{-}(r) \text { are reflections of } R^{+}(r) \text { and } D^{+}(r), \\
& \text { respectively, in } R(r) \text { about the plane } t=t_{0}-\frac{1}{4} r^{2}, \\
& R^{*}(r) \text { is a cylinder containing } R^{-} \text {and contained within } D^{-} .
\end{aligned}
$$

We will need the following information concerning weak subsolutions (supersolutions) of (19). It follows from the general theory as developed in [LSU and T2] that weak subsolutions (supersolutions) of (19) are locally bounded above (below). Also, we have the weak Harnack inequality that was first established in [T2, Theorem 4.1]. 
2.1 THEOREM. Let $u \in W^{1,2}(\Omega)$ be a weak subsolution of (19). Then there is a constant $C$ depending only on the structure (1), inf $u$, and $\tau$ in (22) such that

$$
r^{-(n+2)} \int_{R^{*}(r)}[\mu(r)-u(x, t)] d x d t \leq C[\mu(r)-\mu(r / 2)]+m(r)
$$

where $m(r) \rightarrow 0$ as $r \downarrow 0$ and $\mu(r)=\sup \{u(z): z \in R(r)\}$.

Notice that if $u$ is nonnegative,

$$
r^{-(n+2)} \int_{R(r)}[\mu(r)-u(x, t)] d x d t \leq C r^{-(n+2)} \int_{R^{*}(r)}[\mu(r)-u(x, t)] d x d t
$$

where the constant $C$ depends only on the ratio of the volumes $|R(r)| /\left|R^{*}(r)\right|$, because $\int_{R^{*}(r)} u \leq \int_{R(r)} u$. Therefore, it follows from Theorem 2.1 that

$$
f_{R(r)}[\mu(r)-u(x, t)] d x d t \rightarrow 0 \quad \text { as } r \downarrow 0
$$

where we recall that $f$ denotes the integral average. The significance of $(23)$ is that $R(r)$ contains the point $z_{0}$ whereas $R^{*}(r)$ does not. Thus, if we define $\Lambda$ at $z_{0}$ to be $\Lambda\left(z_{0}\right)=\lim _{r \rightarrow 0} \mu(r)$, we can conclude from a result of [JMZ] concerning Lebesgue points defined in terms of irregular sets (parabolic cylinders in our case) that $u(z)=\Lambda(z)$ for almost all $z \in \Omega$. This discussion is based on the assumption that the subsolution $u$ is nonnegative. If $u$ is bounded below by $k$, we arrive at the same conclusions because the function $u-k$ is a nonnegative subsolution of another equation with structure (1). Observing that $\Lambda$ is an upper semicontinuous function, we have the following result.

2.2 THEOREM. If $u \in W^{1,2}(\Omega)$ is a weak subsolution (supersolution) of (19) that is bounded below (above), then

$$
\begin{aligned}
\lim _{r \rightarrow 0} & f_{R(r)}\left|u(x, t)-u\left(x_{0}, t_{0}\right)\right| d x d t \\
& =\lim _{r \rightarrow 0} f_{R(r)}|\mu(r)-u(x, t)| d x d t=0
\end{aligned}
$$

for all $z_{0}=\left(x_{0}, t_{0}\right) \in \Omega$. In case $u$ is a supersolution, $\mu(r)$ must be replaced by $\lambda(r)=\inf \{u(z): z \in R(r)\}$. Thus, weak subsolutions (supersolutions) of (19) are defined at all points of $\Omega$ and are upper (lower) semicontinuous.

We now recall the concept of thermal capacity, $\operatorname{cap}_{T}$, that was defined in (14). In terms of it, we define a set $A \subset R^{n+1}$ to be thin at $z_{0}$ if

$$
\int_{0}^{1} \frac{\operatorname{cap}_{T}\left[R^{-}(r) \cap A\right]}{\operatorname{cap}_{T}\left[R^{-}(r)\right]} \frac{d r}{r}<\infty
$$

where $R^{-}(r)$ is described in (22). We will need the following lemma later in the development.

2.3 LEMMA. If $\left\{A_{i}\right\}$ is a sequence of sets each of which is thin at $z_{0}$, then there exists a sequence of real numbers $\left\{r_{i}\right\}$ such that $\bigcup_{i=1}^{\infty} A_{i} \cap R^{-}\left(r_{i}\right)$ is thin at $z_{0}$.

Proof. Because $A_{i}$ is thin at $z_{0}$, it follows that

$$
\lim _{r \rightarrow 0} \inf \frac{\operatorname{cap}_{T}\left[R^{-}(r) \cap A_{i}\right]}{\operatorname{cap}_{T}\left[R^{-}(r)\right]}=0 \text {. }
$$


For each $i$, choose $r_{i}$ such that

$$
\int_{0}^{r_{i}} \frac{\operatorname{cap}_{T}\left[A_{i} \cap R^{-}(r)\right]}{\operatorname{cap}_{T}\left[R^{-}(r)\right]} \frac{d r}{r}<2^{-(i+1)}
$$

Then,

$$
\begin{aligned}
\int_{0}^{1} & \frac{\operatorname{cap}_{T}\left[A_{i} \cap R^{-}\left(r_{i}\right) \cap R^{-}(r)\right]}{\operatorname{cap}_{T}\left[R^{-}(r)\right]} \frac{d r}{r} \\
= & \int_{0}^{r_{i}} \frac{\operatorname{cap}_{T}\left[A_{i} \cap R^{-}\left(r_{i}\right) \cap R^{-}(r)\right]}{\operatorname{cap}_{T}\left[R^{-}(r)\right]} \frac{d r}{r} \\
& +\int_{r_{i}}^{1} \frac{\operatorname{cap}_{T}\left[A_{i} \cap R^{-}\left(r_{i}\right) \cap R^{-}(r)\right]}{\operatorname{cap}_{T}\left[R^{-}(r)\right]} \frac{d r}{r} \\
< & 2^{-(i+1)}+C \operatorname{cap}_{T}\left[A_{i} \cap R^{-}\left(r_{i}\right)\right]\left[r_{i}^{-n}-1\right] \\
< & 2^{-i} \quad \text { for } r_{i} \operatorname{sufficiently~small.~}^{-n}
\end{aligned}
$$

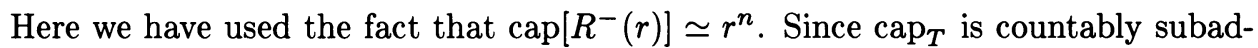
ditive, the result now easily follows.

3. The main results. We now consider a solution $u \in W^{1,2}(\Omega)$ to the obstacle problem as described in (15) and (16). Thus, the obstacle $\psi$ is assumed to be defined q.e. on $\Omega$ and is bounded above. Also, the solution $u$ is a supersolution of (19). Our first result states that $u$ behaves like a subsolution when truncated below away from the obstacle.

3.1 THEOREM. Suppose there is a constant $M$ such that $\psi(z) \leq M$ for all $z \in R(r)$ where $r \leq 1$ and is small enough so that $R(r) \subset \Omega$. Then, there is a constant $C$ depending only on the structure (1) and $\tau$ in (21) such that

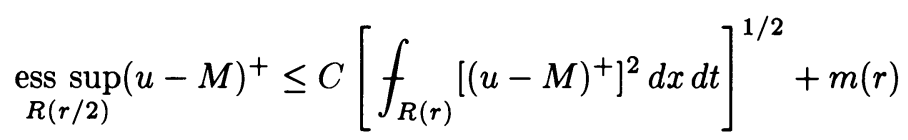

where $m(r) \rightarrow 0$ as $r \rightarrow 0$.

ProOF. Let $v=(u-M)^{+}$and for $k \geq 0$, define $w=(v-k)^{+}$. Now define a test function $\varphi$ by $\varphi(z)=-w(z) \eta(z)^{2}$ where $\eta(x, t) \in C_{0}^{\infty}\left(R(r)_{t}\right)$. Note that

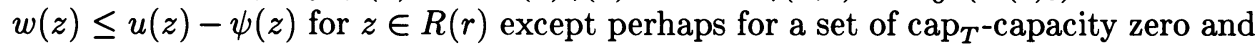
therefore it follows that $\varphi$ is an admissible test function for (16). Consequently,

$$
\iint_{R(r)} u_{t} \varphi+A\left(x, t, u, u_{x}\right) \cdot \nabla \varphi-B\left(x, t, u, u_{x}\right) \varphi \geq 0
$$

Now

and

$$
\iint_{R(r)} u_{t} \varphi=\iint_{R(r)} w_{t} \varphi
$$

$$
\nabla \varphi=-2 w \eta \nabla \eta-\eta^{2} \nabla u \chi_{\{u>M+k\}}
$$

where $\chi_{A}$ denotes the characteristic function of a set $A$. Therefore (25) takes the form

$$
\iint_{R(r) \cap\{u \geq M+k\}} w_{t} \varphi-\eta^{2} A \cdot \nabla u-2 w \eta A \cdot \nabla \eta-B w \eta^{2} \geq 0 .
$$


Using the structure (1), we then have

$$
\begin{aligned}
\iint_{R(r) \cap} & \{u \geq M+k\} \\
\leq & \iint_{R(r) \cap\{u \geq M+k\}} \eta_{1}^{2} w w_{t}+\eta^{2}|\nabla w|^{2} d x d t \\
& +\iint_{R(r) \cap\{u \geq M+k\}} 2 w \eta|\nabla \eta|\left[a_{0}|\nabla w|+a_{1}|u|+a_{2}\right] d x d t \\
& +c_{2} \iint_{R(r) \cap\{u \geq M+k\}} \eta^{2} d x d t .
\end{aligned}
$$

This can be written as

$$
\begin{aligned}
\iint_{R(r)} \cap\{w \geq 0\} & \eta^{2} w w_{t}+\eta^{2}|\nabla w|^{2} d x d t \\
\leq & \iint_{R(r) \cap\{w \geq 0\}} \eta^{2} w\left[b_{1}|\nabla w|+b_{2}|w|+b_{2}(1+M+k)+b_{3}\right] d x d t \\
& \left.+\iint_{R(r) \cap\{w \geq 0\}} 2 w \eta|\nabla \eta|\left|a_{0}\right| \nabla w\left|+a_{1}\right| w \mid+a_{1}(1+M+k)+a_{2}\right] d x d t \\
& +\iint_{R(r) \cap\{w \geq 0\}} \eta^{2}\left[4 c_{1}|w|^{2}+8 c_{1}\left(1+M^{2}+k^{2}\right)+c_{2}\right] d x d t .
\end{aligned}
$$

After several applications of Young's inequality, this becomes

$$
\begin{aligned}
& \iint_{R(r) \cap\{w \geq 0\}} \eta^{2} w w_{t}+\frac{1}{3} \eta^{2}|\nabla w|^{2} d x d t \\
& \leq \leq C_{1} \iint_{R(r) \cap\{w \geq 0\}} w^{2}\left(\eta^{2}+|\nabla \eta|^{2}\right) d x d t \\
& \quad+C_{2}\left(k^{2}+\lambda\right) \iint_{R(r) \cap\{w \geq 0\}} \eta^{2} d x d t
\end{aligned}
$$

where $C_{1}, C_{2}$, and $\lambda$ are constants that depend only on the coefficients in the structure (1) while $\lambda$ depends also on $M$.

Let us now assume that the cut off function $\eta$ vanishes in a neighborhood of $\tau_{1}=t_{0}-\frac{3}{4} r^{2}$ and $\tau_{2}=t_{0}+\frac{1}{4} r^{2}$. Then it follows from (28) that

$$
\begin{aligned}
\int_{\tau_{1}}^{\tau_{2}} \int \eta^{2}|\nabla w|^{2} d x d t \leq & K \int_{\tau_{1}}^{\tau_{2}} \int w^{2}\left(\eta^{2}+|\nabla \eta|^{2}\right) d x d t \\
& +C\left(k^{2}+\lambda\right) \int_{\tau_{1}}^{\tau_{2}} \int \eta^{2} d x d t \\
& +C \int_{\tau_{1}}^{\tau_{2}} \int w^{2} \eta\left|\eta_{t}\right| d x d t .
\end{aligned}
$$

Choose $t^{*} \in\left(t_{0}-\frac{3}{4} r^{2}, t_{0}+\frac{1}{4} r^{2}\right)$ so that

$$
\int \eta^{2} w^{2}\left(x, t^{*}\right) d x>\frac{1}{2} \sup \int \eta^{2} w^{2}(x, t) d x
$$


where the sup is taken over all $t \in\left(t_{0}-\frac{3}{4} r^{2}, t_{0}+\frac{1}{4} r^{2}\right)$. If the test function $\varphi$ is replaced by $\chi_{\left[t_{0}-3 r^{2} / 4, t^{*}\right]} \varphi$ (see (16) and (17)), one obtains from (28) that

$$
\sup _{t} \int \eta^{2} w^{2}(x, t) d x+\int_{\tau_{1}}^{t^{*}} \int \eta^{2}|\nabla w|^{2} d x d t
$$

is dominated by the right side of (29). Therefore, recalling that $w=(v-k)^{+}$,

$$
\begin{aligned}
\sup _{t} \int & \eta^{2}\left[(v-k)^{+}\right]^{2}(x, t) d x+\int_{\tau_{1}}^{\tau_{2}} \int \eta^{2}\left|\nabla(v-k)^{+}\right|^{2} d x d t \\
\leq & C_{1} \int_{\tau_{1}}^{\tau_{2}} \int(v-k)^{+2}\left(\eta^{2}+|\nabla \eta|^{2}\right) d x d t \\
& +C_{2}\left(k^{2}+\lambda\right) \int_{\tau_{1}}^{\tau_{2}} \int_{\{v>k\}_{t}} \eta^{2} d x d t \\
& +C \int_{\tau_{1}}^{\tau_{2}} \int(v-k)^{+2} \eta\left|\eta_{t}\right| d x d t .
\end{aligned}
$$

We now wish to make a change of variable in (31) that will have the effect of causing the term that involves $\lambda$ to vanish. For this purpose, we will first rescale (31) with the result that the basic parabolic cylinder $R(r)$ is replaced by $R(1)$. Thus, if we define $\bar{v}(x, t)=v\left(x_{0}+r x, t_{0}+r^{2} t\right) \cdot r^{-1}$ and $\bar{\eta}$ similarly, then $\bar{v}$ and $\bar{\eta}$ will satisfy (31) in $R(1)$ provided $r \leq 1$. Rather than invoking the notation $\bar{v}$, and $\bar{\eta}$, we will now assume that (31) has been rescaled and all integrals are taken over $R(1)$.

We now will proceed to reformulate (31) so that $\lambda$ is eliminated. Let $\Lambda=2 \lambda^{1 / 2}$ and define $\bar{v}=v+\Lambda$. There are two cases to consider.

Case 1. $k \geq \Lambda$. Using (31) with $k$ replaced by $k-\Lambda$, we have

$$
\begin{aligned}
\sup _{t} \int & \eta^{2}(\bar{v}-k)^{+2}(x, t) d x+\int_{\tau_{1}}^{\tau_{2}} \int \eta^{2}\left|\nabla(\bar{v}-k)^{+}\right|^{2} d x d t \\
= & \sup _{t} \int_{\tau^{2}} \eta^{2}\left(v-(k-\Lambda)^{+2}(x, t) d x\right. \\
& +\int_{\tau_{2}}^{\tau_{2}} \int \eta^{2}\left|\nabla(v-(k-\Lambda))^{+}\right|^{2} d x d t \\
\leq & C \int_{\tau_{1}}^{\tau_{2}} \int(v-(k-\Lambda))^{+2}\left(\eta^{2}+|\nabla \eta|^{2}\right) d x d t \\
& +C\left((k-\Lambda)^{2}+\lambda\right) \int_{\tau_{1}}^{\tau_{2}} \int_{\{v>(k-\Lambda)\}_{t}} \eta^{2} d x d t \\
& +C \int_{\tau_{1}}^{\tau_{2}} \int(v-(k-\Lambda))^{+2} \eta\left|\eta_{t}\right| d x d t \\
\leq & C \int_{\tau_{1}}^{\tau_{2}} \int(\bar{v}-k)^{+2}\left(\eta^{2}+|\nabla \eta|^{2}\right) d x d t \\
& +C\left(k^{2}-\Lambda^{2}+\lambda\right) \int_{\tau_{1}}^{\tau_{2}} \int \\
& +C \int_{\{\bar{v}>k\}_{t}}^{\tau_{2}} \int(\bar{v}-k)^{+2} \eta\left|\eta_{t}\right| d x d t .
\end{aligned}
$$


Observe that $k^{2}-\Lambda^{2}+\lambda<k^{2}$ and therefore

$$
\begin{aligned}
\sup _{t} \int & \eta^{2}(\bar{v}-k)^{+2}(x, t) d x+\int_{\tau_{1}}^{\tau_{2}} \int \eta^{2}\left|\nabla(\bar{v}-k)^{+}\right|^{2} d x d t \\
\leq & C \int_{\tau_{1}}^{\tau_{2}} \int(\bar{v}-k)^{+2}\left(\eta^{2}+|\nabla \eta|^{2}\right) d x d t \\
& +C k^{2} \int_{\tau_{1}}^{\tau_{2}} \int_{\{\bar{v}>k\}_{t}} \eta^{2} d x d t \\
& +C \int_{\tau_{1}}^{\tau_{2}} \int(\bar{v}-k)^{+2} \eta\left|\eta_{t}\right| d x d t
\end{aligned}
$$

whenever $k \geq 0$. This yields the desired result in this case.

Case 2. $0 \leq k<\Lambda$. From (31),

$$
\begin{aligned}
\sup _{t} \int & \eta^{2}\left[(\bar{v}-k)^{+}\right]^{2}(x, t) d x+\int_{\tau_{1}}^{\tau_{2}} \int \eta^{2}\left|\nabla(\bar{v}-k)^{+}\right|^{2} d x d t \\
= & \sup _{t} \int \eta^{2}\left[(\bar{v}-0)^{+}\right]^{2}(x, t) d x+\int_{\tau_{1}}^{\tau_{2}} \int \eta^{2}\left|\nabla(v-0)^{+}\right|^{2} d x d t \\
\leq & C \int_{\tau_{1}}^{\tau_{2}} \int\left[(v-0)^{+}\right]^{2}\left(\eta^{2}+|\nabla \eta|^{2}\right) d x d t+C \lambda \int_{\tau_{1}}^{\tau_{2}} \int_{\{v>0\}_{t}} \eta^{2} d x d t \\
& +C \int_{\tau_{1}}^{\tau_{2}} \int\left[(v-0)^{+}\right]^{2} \eta\left|\eta_{t}\right| d x d t \\
= & C \int_{\tau_{1}}^{\tau_{2}} \int(v-(k-\Lambda))^{+2}\left(\eta^{2}+|\nabla \eta|^{2}\right) d x d t+C \lambda \int_{\tau_{1}}^{\tau_{2}} \int_{\{v>k-\Lambda\}_{t}} \eta^{2} d x d t \\
& +C \int_{\tau_{1}}^{\tau_{2}} \int(v-(k-\Lambda))^{+2} \eta\left|\eta_{t}\right| d x d t \\
= & C \int_{\tau_{1}}^{\tau_{2}} \int(\bar{v}-k)^{+2}\left(\eta^{2}+|\nabla \eta|^{2}\right) d x d t+C \lambda \int_{\tau_{1}}^{\tau_{2}} \int_{\{\bar{v}>k\}_{t}} \eta^{2} d x d t \\
& +C \int_{\tau_{1}}^{\tau_{2}} \int(\bar{v}-k)^{+2} \eta\left|\eta_{t}\right| d x d t .
\end{aligned}
$$

If $\frac{1}{2} \Lambda \leq k \leq \Lambda$, then $\lambda=\left(\frac{1}{2} \Lambda\right)^{2} \leq k^{2}$ and we have achieved the desired form (32). If $0 \leq k \leq \frac{1}{2} \Lambda$, then $\bar{v}(z)-k>\frac{1}{2} \Lambda$ for all $z \in R(1)$. Hence, $(\bar{v}(z)-\bar{k})^{+2}>\lambda$, and again the desired inequality (32) follows.

We now wish to put (32) into a form that will be suitable to perform a Moser-type iteration. This will then lead to the conclusion of the theorem.

To this end, rewrite (32) as

$$
\begin{aligned}
\sup _{t} & \int_{\{\bar{v}>k\}_{t}}\left(\eta^{2}\left(\bar{v}^{2}-2 \bar{v} k+k^{2}\right)\right) d x+\int_{\tau_{1}}^{\tau_{2}} \int \eta^{2}\left|\nabla(\bar{v}-k)^{+}\right|^{2} d x d t \\
\leq & C \int_{\tau_{1}}^{\tau_{2}} \int_{\{\bar{v}>k\}_{t}}\left(\eta^{2}+|\nabla \eta|^{2}\right)\left(\bar{v}^{2}-2 \bar{v} k+k^{2}\right) d x d t \\
& +C \int_{\tau_{1}}^{\tau_{2}} \int_{\{\bar{v}>k\}_{t}} \eta\left|\eta_{t}\right|\left(\bar{v}^{2}-2 \bar{v} k+k^{2}\right) d x d t .
\end{aligned}
$$


For $\beta>1$, multiply both sides of (34) by $k^{\beta-2}$ and integrate with respect to $k$. By Fubini's theorem we have

$$
\begin{gathered}
C(\beta) \sup _{t} \int \eta^{2} \bar{v}^{\beta+1}+\frac{1}{\beta-1} \iint \eta^{2} \bar{v}^{\beta-1}|\nabla \bar{v}|^{2} d x d t \\
\leq C C(\beta) \iint\left(\eta^{2}+|\nabla \eta|^{2}\right) \bar{v}^{\beta+1} d x d t \\
\quad+C C(\beta) \iint \eta\left|\eta_{t}\right| \bar{v}^{\beta+1} d x d t
\end{gathered}
$$

where

$$
C(\beta)=\frac{1}{\beta-1}-\frac{2}{\beta}+\frac{1}{\beta+1} .
$$

This implies that

$$
\begin{aligned}
& \frac{1}{\beta+1} \sup _{t} \int \eta^{2} \bar{v}^{\beta+1} d x+\iint \eta^{2} \bar{v}^{\beta-1}|\nabla \bar{v}|^{2} d x d t \\
& \leq C \iint\left(\eta^{2}+|\nabla \eta|^{2}\right) \bar{v}^{\beta+1} d x d t+C \iint \eta\left|\eta_{t}\right| \bar{v}^{\beta+1} d x d t .
\end{aligned}
$$

We now appeal to a well-known iteration scheme cf. [T], to conclude from (35) that

$$
\underset{R(1 / 2)}{\operatorname{ess} \sup } \bar{v} \leq C\left[\int_{R(1)} \bar{v}^{2} d x d t\right]^{1 / 2} .
$$

Recalling that $\bar{v}=v+\Lambda$ and $v=(u-M)^{+}$, the conclusion of the theorem follows by rescaling.

3.2 REMARK. The reader will have no difficulty in verifying that Theorem 3.1 remains valid if the notion of the solution to the obstacle problem is taken in the sense used by $[\mathbf{M P}, \mathbf{P}]$, or $[\mathbf{B M}]$ as discussed in $(18), \S 1$. With this notion of solution, if one considers the test function $\varphi=\eta^{2}[u-(M+k)]^{+}$, then (25) becomes an equality and the remainder of the argument remains essentially unchanged.

We now will establish the fine continuity of subsolutions of (19) by employing a technique introduced in [GZ2].

3.3 THEOREM. Let $u \in W^{1,2}(\Omega)$ be a bounded weak subsolution (supersolution) of (19). Then for each $z_{0} \in \Omega$ and $\varepsilon>0$, the set

$$
\Omega \cap\left\{z: u(z)<u\left(z_{0}\right)-\varepsilon\right\} \quad\left(\Omega \cap\left\{z: u(z)>u\left(z_{0}\right)+\varepsilon\right\}\right)
$$

is thin at $z_{0}$.

PROOF. We will only consider the case in which $u$ is a subsolution, the other case being treated by an analogous argument.

Because $u$ is a subsolution, we have from Theorem 2.2 that $u$ is upper semicontinuous and therefore the set

$$
E\left(z_{0}, \varepsilon\right)=\Omega \cap\left\{z: u(z)<u\left(z_{0}\right)-\varepsilon\right\}
$$

is open. Consider the parabolic cylinder $R(r)$ as described in (21) and let $K$ be any compact subset of $R^{-}(r) \cap E\left(z_{0}, \varepsilon\right)$. Consider the thermal capacity of $K, \operatorname{cap}_{T}(K)$, as defined in (14) and let $\nu$ denote the equilibrium measure of $K$. Let $\zeta=E * \nu$ denote the corresponding equilibrium potential. Let $\rho: R^{n+1} \rightarrow R^{1}$ be a smooth 
function with compact support such that $\int \rho(z) d z=1$. With $\rho$ as a modifier, let $\nu_{\delta}=\rho_{\delta} * \nu$ and $\zeta \delta=E * \nu_{\delta}$. Since $R^{-}(r) \cap E\left(z_{0}, \varepsilon\right)$ is open we may choose $\delta>0$ so small that

$$
\text { spt } \nu_{\delta} \subset R^{-}(r) \cap E\left(z_{0}, \varepsilon\right) \text {. }
$$

Let $1<\tau_{1}<\tau_{2}<4 / 3$ and let $\eta$ be a smooth cutoff function such that spt $\eta \subset$ $B\left(x_{0}, \tau_{2}(r / 2)\right), \eta \equiv 1$ on $B\left(x_{0}, \tau_{1}(r / 2)\right)$ and $|\nabla \eta| \leq C / r_{0}$. We will consider $\eta$ as defined on $R^{n+1}$, independent of $t$.

Fix $t_{1}$ such that $t_{0}-\frac{3}{4} r^{2}<t_{1}<t_{0}-\frac{9}{16} r^{2}$ where $t_{0}-\frac{9}{16} r^{2}$ is the lower $t$-coordinate of $R^{-}(r / 2)$. We may choose $\delta$ sufficiently small so that

$$
\varsigma_{\delta}(x, t)=0 \quad \text { for } t \leq t_{1} .
$$

Let $w=\left[u-\left(u\left(z_{0}\right)-\varepsilon\right)\right]^{+}$and define $f(w)=\mu(r)-w^{2}$, where $\mu(r)=\sup \{w(z): z \in$ $R(r)\}$. Note from Theorem 2.1 that $\mu(r) \rightarrow \varepsilon$ as $r \downarrow 0, w=0$ on $E\left(z_{0}, \varepsilon\right)$, and that $f(w) \geq \varepsilon$ on $E\left(z_{0}, \varepsilon\right)$.

Let $H=\partial / \partial t-\Lambda$ denote the heat operator. For almost all $t_{2} \in\left(t_{1}, t_{0}-\frac{1}{4} r^{2}\right)$, we have that

(38)

$$
\begin{aligned}
\int_{t_{1}}^{t_{2}} \int & \left(H_{\zeta}\right)(\eta f(w)) d x d t=\int_{t_{1}}^{t_{2}} \int{ } \eta f(w) d x d t+\int_{t_{1}}^{t_{2}} \int \nabla_{\zeta} \cdot \nabla(\eta f(w)) d x d t \\
= & \int \varsigma \eta f(w)\left(x, t_{2}\right) d x-\int_{t_{1}}^{t_{2}} \int \varsigma(\eta f(w))_{t} d x d t \\
& +\int_{t_{1}}^{t_{2}} \int \nabla \zeta \cdot \nabla(\eta f(w)) d x d t \int \varsigma \eta f(w)\left(x, t_{2}\right) d x \\
& -\int_{t_{1}}^{t_{2}} \int \eta \zeta f^{\prime}(w) w_{t} d x d t+\int_{t_{1}}^{t_{2}} \int \nabla_{\zeta} \cdot \nabla(\eta f(w)) d x d t
\end{aligned}
$$

because $\eta$ is a function of $x$ only.

Now define a test function $\varphi=\eta \zeta f^{\prime}(w) \in W_{0}^{1,2}\left(\Omega_{t}\right)$. Then, because $u$ is a subsolution and $\varphi \leq 0$,

$$
\int_{t_{1}}^{t_{2}} \int\left(\eta \zeta f^{\prime}(w)\right) u_{t}+A \cdot \nabla \varphi-B \varphi \geq 0
$$

or

$$
\begin{gathered}
-\int_{t_{1}}^{t_{2}} \int \eta_{\zeta} f(w)_{t} d x d t-\int_{t_{1}}^{t_{2}} \int A(x, t, u, \nabla u) \cdot \nabla\left(\eta_{\zeta} f^{\prime}(w)\right) d x d t \\
\leq-\int_{t_{1}}^{t_{2}} \int B \cdot\left(\eta_{\zeta} f^{\prime}(w)\right) d x d t
\end{gathered}
$$

that is,

$$
\begin{aligned}
& -\int_{t_{1}}^{t_{2}} \int \eta \zeta f^{\prime}(w) w_{t} d x d t-\int_{t_{1}}^{t_{2}} \int(\eta \zeta) A \cdot \nabla w f^{\prime \prime}(w)+A \cdot \nabla(\eta \zeta) f^{\prime}(w) d x d t \\
& \leq-\int_{t_{1}}^{t_{2}} \int B \cdot\left(\eta \zeta f^{\prime}(w)\right) d x d t .
\end{aligned}
$$


Because we have assumed that $u$ is bounded, we may use the structure (1) with the understanding that the terms involving $|u|$ are incorporated in the constants $a_{2}, b_{2}$, and $c_{2}$. Thus, we have

$$
\begin{gathered}
-\int_{t_{1}}^{t_{2}} \int \eta \zeta f^{\prime}(w) w_{t} d x d t+c \int_{t_{1}}^{t_{2}} \int(\eta \zeta)\left[|\nabla u|^{2}-c_{2}\right] d x d t \\
-2 \int_{t_{1}}^{t_{2}} \int w A \cdot \nabla(\eta \zeta) d x d t \\
\leq-\int_{t_{1}}^{t_{2}} \int B \cdot\left(\eta \zeta f^{\prime}(w)\right) d x d t
\end{gathered}
$$

Thus,

$$
\begin{aligned}
-\int_{t_{1}}^{t_{2}} & \int \eta \zeta f^{\prime}(w) w_{t} d x d t+c \int_{t_{1}}^{t_{2}} \int(\eta \zeta)\left[|\nabla w|^{2}-c_{2}\right] d x d t \\
\leq & \int_{t_{1}}^{t_{2}} \int w\left[|\nabla w|+a_{2}\right]|\nabla(\eta \zeta)| d x d t \\
& +\int_{t_{1}}^{t_{2}} \int\left[|\nabla w|+b_{2}\right]\left|\eta \zeta f^{\prime}(w)\right| d x d t \\
\leq & C \int_{t_{1}}^{t_{2}} \int \eta w\left[|\nabla w|+a_{2}\right]|\nabla \zeta| d x d t+C \iint \varsigma w\left[|\nabla w|+a_{2}\right]|\nabla \eta| d x d t \\
& +C \int_{t_{1}}^{t_{2}} \int\left[|\nabla w|+b_{2}\right][\eta \zeta w] d x d t .
\end{aligned}
$$

Hence, by several applications of Young's inequality and using the fact that $w$ is bounded,

$$
\begin{aligned}
&-\int_{t_{1}}^{t_{2}} \int \eta \zeta f^{\prime}(w) w_{t} d x d t+\frac{c}{2} \int_{t_{1}}^{t_{2}} \int(\eta \zeta)|\nabla w|^{2} d x d t \\
& \leq \frac{\varepsilon}{4} \int_{t_{1}}^{t_{2}} \int|\nabla \zeta|^{2} d x d t+C_{\varepsilon}(w) \int_{t_{1}}^{t_{2}} \int \eta^{2}\left[|\nabla w|+a_{2}\right]^{2} d x d t \\
&+C(w) \int_{t_{1}}^{t_{2}} \int \zeta|\nabla w||\nabla \eta| d x d t+C_{\varepsilon}(w) \int_{t_{1}}^{t_{2}} \int \eta^{2}|\nabla w|^{2} d x d t \\
&+C(w) \int_{t_{1}}^{t_{2}} \int \eta^{2} w^{2} d x d t+C(w) \int_{t_{1}}^{t_{2}} \int \eta \zeta+|\nabla \eta| d x d t .
\end{aligned}
$$

Also,

$$
\int[\varsigma \eta f(w)]\left(x, t_{2}\right) d x \leq \frac{\varepsilon}{4} \int \varsigma^{2}\left(x, t_{2}\right) d x+C_{\varepsilon} \int\left[\eta^{2} f(w)^{2}\right]\left(x, t_{2}\right) d x
$$

and

$$
\begin{aligned}
\int_{t_{1}}^{t_{2}} \int & \nabla \zeta \cdot \nabla(\eta f(w)) d x d t \\
& \leq \frac{\varepsilon}{4} \int_{t_{1}}^{t_{2}} \int|\nabla \zeta|^{2} d x d t+C_{\varepsilon} \int_{t_{1}}^{t_{2}} \int|\nabla(\eta f(w))|^{2} d x d t \\
& \leq \frac{\varepsilon}{4} \int_{t_{1}}^{t_{2}} \int|\nabla \zeta|^{2} d x d t+C_{\varepsilon} \int_{t_{1}}^{t_{2}} \int[|\nabla \eta| f(w)]^{2}+[2 w \eta|\nabla w|]^{2} d x d t .
\end{aligned}
$$


Hence, from (38), (40), (39) and (41),

$$
\begin{aligned}
\iint( & \left.H_{\zeta}\right)(\eta f(w)) d x d t \\
\leq & \frac{\varepsilon}{2}\left[\int \varsigma^{2}\left(x, t_{2}\right) d x+\int_{t_{1}}^{t_{2}} \int|\nabla \zeta|^{2} d x d t\right] \\
& +C(\varepsilon, w)\left[\int_{t_{1}}^{t_{2}} \int \eta^{2}|\nabla w|^{2}+\int_{t_{1}}^{t_{2}} \int \varsigma|\nabla \eta||\nabla u|\right]+C \int_{t_{1}}^{t_{2}} \int \eta \zeta+|\nabla \eta| \\
& +\int \eta^{2} f(w)^{2}\left(x, t_{2}\right) d x+C(w) \int_{t_{1}}^{t_{2}} \int \eta^{2} d x d t \\
& +C(w) \int_{t_{1}}^{t_{2}} \int \eta^{2} w^{2} d x d t+\int_{t_{1}}^{t_{2}} \int|\nabla \eta|^{2} f(w)^{2} d x d t .
\end{aligned}
$$

We now consider all but the first two terms in the above inequality; that is, all terms whose coefficients are not $\varepsilon / 2$. We will determine the rate at which these terms approach zero as $r \downarrow 0$. All of these terms except those involving $|\nabla u|,|\nabla w|$, and the last term are $O\left(r^{n+1}\right)$. In fact, they are all $O\left(r^{n+2}\right)$ except the term involving $|\nabla \eta|$. The remaining terms, $\left[\int_{t_{1}}^{t_{2}} \int \eta^{2}|\nabla w|^{2}, \int_{t_{1}}^{t_{2}} \int \zeta|\nabla \eta||\nabla u|\right]$, and $\int_{t_{1}}^{t_{2}} \int|\nabla \eta|^{2} f(w)^{2}$ can be estimated by technique involving the weak Harnack inequality [T2]. This technique was one of the main contributions of [GZ, Theorem $2.1]$ in the elliptic case and of $[\mathbf{Z}$, Theorem 4.2] in the parabolic case. Thus, it follows there are constants $C$ and $A$ depending only on the structure (1) and the bound for $u$ such that the essential supremum over $t_{2} \in\left(t_{1}, t_{1}+\frac{5}{16} r^{2}\right)$ of these terms is bounded above by

$$
C[\mu(r)-\mu(r / 2)+a r] r^{n} .
$$
that

Next, we estimate the terms involving $\varepsilon$ in (41). Using the fact that $\zeta_{\delta} \leq 1$, note

$$
\begin{aligned}
\operatorname{Cap}_{T}(K) & =\nu_{\delta}\left(R^{n+1}\right) \geq \int_{t_{1}}^{t_{2}} \int\left(H_{\zeta \delta}\right) \zeta_{\delta} d x d t \\
& =\frac{1}{2} \int \varsigma_{\delta}^{2}\left(x, t_{2}\right) d x+\int_{t_{1}}^{t_{2}} \int|\nabla \zeta \delta|^{2} d x d t .
\end{aligned}
$$

Moreover, since $\eta \equiv 1$ on a neighborhood of $K$, we have for all small $\delta>0$,

$$
\begin{aligned}
\int_{t_{1}}^{t_{2}} \int\left(H_{\zeta \delta}\right)(\eta f(w)) d x d t & \geq \int_{t_{1}}^{t_{2}} \int\left(H_{\zeta \delta}\right)\left(\eta\left(\mu(r)-w^{2}\right)\right) d x d t \\
& \geq \mu(r) \operatorname{cap}_{T}(K)-\int_{t_{1}}^{t_{2}} \int\left(H_{\zeta \delta}\right)\left(\eta w^{2}\right) d x d t .
\end{aligned}
$$

Now $H_{\zeta \delta}=\nu_{\delta}$ and $\delta$ was chosen so small that spt $\nu_{\delta} \subset E\left(z_{0}, \varepsilon\right)$. But $w \equiv 0$ on $E\left(z_{0}, \varepsilon\right)$ and therefore the last integral above is zero. Hence, from (44),

$$
\int_{t_{1}}^{t_{2}} \int\left(H_{\zeta \delta}\right)(\eta f(w)) d x d t \geq \varepsilon \operatorname{cap}_{T}(K) .
$$

Thus, from (42), (43), and (45)

$$
(\varepsilon / 2) \operatorname{cap}_{T}(K) \leq C[\mu(r)-\mu(r / 2)+a r] r^{n} .
$$


Because $K$ is an arbitrary compact subset of $R^{-}(r) \cap E\left(z_{0}, \varepsilon\right)$ and because of the inner regularity of thermal capacity, it follows from (46) that

$$
\frac{\varepsilon}{2} \frac{\operatorname{cap}_{T}\left[R^{-}(r) \cap E\left(z_{0}, \varepsilon\right)\right]}{r^{n}} \leq C\left[\mu(r)-\mu\left(\frac{r}{2}\right)+a r\right] .
$$

It is a simple matter to verify that

$$
\int_{0}^{1}\left[\mu(r)-\mu\left(\frac{r}{2}\right)+a r\right] \frac{d r}{r}<\infty,
$$

and thus, the proof is complete.

3.4 DEFINITION. A function $u: \Omega \rightarrow R^{1}$ is said to be finely continuous at $z_{0} \in \Omega$ if there is a set $E$ such that $E$ is thin at $z_{0}$ and

$$
\lim _{\substack{z \rightarrow z_{0} \\ z \in(\Omega-E) \cap R^{-}}} u(z)=u\left(z_{0}\right) \text { where } R^{-}=\bigcup_{r>0} R^{-}(r) .
$$

3.5 COROLlaRY. Let $u \in W^{1,2}(\Omega)$ be a bounded weak subsolution or supersolution of (19). Then $u$ is finely continuous at each point $z_{0} \in \Omega$.

ProOF. Consider the case where $u$ is a subsolution. From Theorem 3.3 we have that $\Omega \cap\left\{z: u(z)<u\left(z_{0}\right)-\varepsilon\right\}$ is thin for each $\varepsilon>0$. Let $\left\{\varepsilon_{i}\right\}$ be a decreasing sequence tending to 0 and let $E_{i}=\Omega \cap\left\{z: u(z)<u\left(z_{0}\right)-\varepsilon_{i}\right\}$. From Lemma 2.3 there is a sequence of real numbers $\left\{r_{i}\right\} \rightarrow 0$ such that $E=\bigcup_{i=1}^{\infty} E_{i} \cap R^{-}\left(r_{i}\right)$ is thin at $z_{0}$. Now suppose that $z_{i} \rightarrow z_{0}, z_{i} \in(\Omega-E) \cap R^{-}$. For each positive integer $j_{0}$ there exists a positive integer $i_{0}$ such that if $i \geq i_{0}$, then $z_{i} \in R^{-}\left(r_{j}\right)$ for some $j \geq j_{0}$. Thus, $z_{i} \notin E_{j}$. This implies that $u\left(z_{i}\right) \geq u\left(z_{0}\right)-\varepsilon_{j} \geq u\left(z_{0}\right)-\varepsilon_{j_{0}}$ for all $i \geq i_{0}$. Consequently,

$$
\liminf _{\substack{z \rightarrow z_{0} \\ z \in(\Omega-E) \cap R^{-}}} u(z) \geq u\left(z_{0}\right)
$$

and the conclusion follows with reference to Theorem 2.2. In case $u$ is a supersolution, apply the preceding argument to $-u$ which is subsolution to an equation of the form (19).

We now discuss the condition on the obstacle $\psi$ that will ensure the continuity of the solution as discussed in the introduction.

3.6 Definition. Let $\psi: R^{n+1} \rightarrow R^{1}$ be defined at all points except perhaps for a set of capacity zero. For $z_{0} \in \Omega$, let $\bar{\psi}(r)=\operatorname{ess} \sup \left\{\psi(z): z \in R\left(z_{0}, r\right)\right\}$ where the ess sup is taken in the sense of capacity. Let $\bar{\psi}\left(z_{0}\right)=\lim _{r \downarrow 0} \bar{\psi}(r)$. We say that $\psi$ is upper regular at $z_{0}$ (in the fine topology) if there is a set $A$ which is not thin at $z_{0}$ such that

$$
\lim _{\substack{z \rightarrow z_{0} \\ z \in A}} \psi(z)=\bar{\psi}\left(z_{0}\right)
$$

3.7 THEOREM. Let $\psi: R^{n+1} \rightarrow R^{1}$ be upper regular at $z_{0} \in \Omega$. Let $u \in$ $W^{1,2}(\Omega)$ be a solution of the obstacle problem (15) and (16). Then, $u$ is continuous at $z_{0}$.

PROOF. Since $u$ is a weak supersolution of (19), $u$ is locally bounded below and from Theorem 3.1 that $u$ is locally bounded above. Thus, from Theorem 2.2 we 
have that $u$ is lower semicontinuous on $\Omega$. Therefore,

$$
\lim _{\substack{z \rightarrow z_{0} \\ z \in \Omega}} \inf u(z) \geq u\left(z_{0}\right)
$$

Also, we conclude from Corollary 3.5 (again using the fact that $u$ is a supersolution) that there is a set $A$ thin at $z_{0}$ with

$$
\lim _{\substack{z \rightarrow z_{0} \\ z \in(\Omega-A)}} \inf u(z)=u\left(z_{0}\right)
$$

In view of (48), if $u$ were not continuous at $z_{0}$, we would have, for some $\varepsilon>0$,

$$
\lim _{\substack{z \rightarrow z_{0} \\ z \in \Omega}} \sup u(z)>u\left(z_{0}\right)+2 \varepsilon .
$$

Because $\psi$ is assumed upper regular at $z_{0}$, it follows that

$$
U(\varepsilon) \equiv\left\{z: \psi(z)>\bar{\psi}\left(z_{0}\right)-\varepsilon\right\}
$$

is not thin at $z_{0}$. Consequently, there is a sequence $\left\{r_{j}\right\} \rightarrow 0$ such that

$$
\mathrm{Cap}_{T}\left[(\Omega-A) \cap U(\varepsilon) \cap R^{-}\left(z_{0}, r_{j}\right)\right]>0
$$

for $j=1,2, \ldots$ Since $u \geq \psi$ everywhere on $\Omega$ except for a set of capacity zero, it follows from (49), (50) and (51) that $u\left(z_{0}\right)>\bar{\psi}\left(z_{0}\right)-\varepsilon$. Choose $r_{0}>0$ and $M$ such that $\bar{\psi}\left(r_{0}\right)<\bar{\psi}\left(z_{0}\right)+\varepsilon$ and $\bar{\psi}\left(r_{0}\right)<M<\bar{\psi}\left(z_{0}\right)+\varepsilon<u\left(z_{0}\right)+2 \varepsilon$. It follows from Theorem 3.1 that

$$
\underset{R(r / 2)}{\operatorname{ess} \sup }(u-M)^{+} \leq C\left[f_{R(r)}\left[(u-M)^{+}\right]^{2} d x d t\right]^{1 / 2}+m(r)
$$

for all $r<r_{0}$. First, suppose that $u\left(z_{0}\right) \leq M$. Since $(u(z)-M)^{+} \leq\left|u(z)-u\left(z_{0}\right)\right|$, it follows from Theorem 2.2 that the right side of (52) tends to zero as $r \downarrow 0$. Therefore,

$$
\lim _{\substack{z \rightarrow z_{0} \\ z \in \Omega}} \sup u(z) \leq M<u\left(z_{0}\right)+2 \varepsilon
$$

contradicting (50). If $u\left(z_{0}\right)>M$, then (52) holds with $M$ replaced by $u\left(z_{0}\right)$ and we conclude ess $\sup _{R(r / 2)}\left(u-u\left(z_{0}\right)\right)^{+} \rightarrow 0$ as $r \downarrow 0$. That is

$$
\lim _{\substack{z \rightarrow z_{0} \\ z \in \Omega}} \sup u(z) \leq u\left(z_{0}\right)
$$

again contradicting (50).

4. Estimates of the modulus of continuity. In this section we will obtain estimates of the modulus of continuity of the solution $u$ in terms of the obstacle $\psi$.

For this purpose, throughout this section we will consider a point $z_{0} \in \Omega$ such that

$$
u\left(z_{0}\right)=\lim _{r \rightarrow 0} \sup \bar{\psi}(r)=\bar{\psi}\left(z_{0}\right)
$$

Let

$$
\begin{array}{r}
\mu(r)=\sup _{R(r)}[u(z)-\bar{\psi}(r)]^{+}, \quad \lambda(r)=\inf _{R(r)}[u(z)-\bar{\psi}(r)]^{+}, \\
\bar{u}(r)=\sup _{R(r)} u, \quad \underline{u}(r)=\inf _{R(r)} u, \quad \bar{\psi}(r)=\text { ess } \sup _{R(r)} \psi,
\end{array}
$$

and define $v(z)=\mu(r)-[u(z)-\bar{\psi}(r)]^{+}$. 
Note that (53) is the only case of interest, for if $u\left(z_{0}\right)>\bar{\psi}\left(z_{0}\right)$, the lower semicontinuity of $u$ would imply, for $r$ sufficiently small and some open set $U$ containing $z_{0}$, that $u(z)>\bar{\psi}(r)$ for all $z \in U$. Then, $u$ becomes a weak solution in $U$ and is therefore Hölder continuous there, cf. [AS, T2].

4.1 LEMMA. There are constants $C$ and $C^{\prime}$ depending only on the structure (1) and $\tau$ in (22) such that

$$
\bar{u}(r)-\bar{u}\left(\frac{r}{2}\right) \geq C f_{R^{*}(r)}\left[\mu(r)-[u(x, t)-\bar{\psi}(r)]^{+}\right] d x d t-C^{\prime} r
$$

for all $r$ such that $R(r) \subset \Omega$.

ProOF. For each number $k$ such that $0 \leq k \leq \mu(r)$, let $w=(v-k)^{-}$and define a test function $\varphi$ by $\varphi=-\eta^{2} w$. Because of the restriction on $k$, it is clear that $w \leq u-\psi$ and therefore $\varphi$ is admissible in (16) whenever $\eta \in C_{0}^{\infty}(R(r))$. The development proceeds exactly as in (25) and (26) leading to (28) with $(v-k)^{+}$ replaced by $(v-k)^{-}$. We wish to reformulate $(28)$ where now $w=(v-k)^{-}$ so that the term involving $\lambda$ does not appear. This proceeds exactly the same way as in the proof of Theorem 3.1. Thus, first rescale (28) by defining $\bar{v}(x, t)=$ $v\left(x_{0}+r x, t_{0}+r^{2}\right) r^{-1}$ and $\bar{\eta}$ similarly. Then $\bar{w}=(\bar{v}-k)^{-}$and $\bar{\eta}$ satisfy (28) in $R(1)$ provided $r \leq 1$. As in the proof of Theorem 3.1, we will not invoke the notation $\bar{v}, \bar{w}$, and $\bar{\eta}$ but rather assume that (28) has been rescaled and all integrals are taken over $R(1)$. Now with the understanding that (28) has been rescaled, let $\bar{v}=v+\Lambda$ and proceed as in Theorem 3.1. We then find

$$
\begin{aligned}
& \iint_{R(1) \cap\{\bar{v}<k\}} \eta^{2}(\bar{v}-k)^{-} \bar{v}_{t} d x d t+\frac{1}{3} \iint_{R(1) \cap\{\bar{v}<k\}} \eta^{2}|\nabla \bar{v}|^{2} d x d t \\
& \leq C \iint_{R(1) \cap\{\bar{v}<k\}}\left[(\bar{v}-k)^{-}\right]^{2}\left(\eta^{2}+|\nabla \eta|^{2}\right) d x d t+C k^{2} \iint_{R(1) \cap\{\bar{v}<k\}} \eta^{2} d x d t
\end{aligned}
$$

Note that $k$ is now required to satisfy $0 \leq k \leq \mu(r) r^{-1}+\Lambda$.

From elementary considerations, we may assume that $\bar{v}>\varepsilon>0$. The results may then be extended by taking $\varepsilon \rightarrow 0$. Let $T>0, \gamma>0$ and put $\bar{v}_{T}=\inf \{T, \bar{v}\}$, $\eta_{T}=\bar{v}_{T}^{\gamma} \eta$. If $\eta_{T}$ is substituted for $\eta$ in (55), we obtain

$$
\begin{aligned}
& -\iint_{R(1) \cap\{\bar{v}<k\}} \bar{v}_{T}^{2 \gamma} \eta^{2}(k-\bar{v}) \bar{v}_{t} d x d t+\frac{1}{3} \iint_{R(1) \cap\{\bar{v}<k\}} \bar{v}_{T}^{2 \gamma} \eta^{2}|\nabla \bar{v}|^{2} d x d t \\
& \leq C \iint_{R(1) \cap\{\bar{v}<k\}}\left[\bar{v}_{T}^{2 \gamma} \eta^{2}+\gamma^{2} \bar{v}_{T}^{2 \gamma-2}\left|\nabla \bar{v}_{T}\right|^{2} \eta^{2}+\bar{v}_{T}^{2 \gamma}|\nabla \eta|^{2}\right]\left[(\bar{v}-k)^{-}\right]^{2} d x d t \\
& \quad+C \iint_{R(1) \cap\{\bar{v}<k\}} \bar{v}_{T}^{2 \gamma} \eta^{2}\left[(\bar{v}-k)^{-}\right]^{2} d x d t+C k^{2} \iint_{R(1) \cap\{\bar{v}<k\}} \bar{v}_{T}^{2 \gamma} \eta^{2} d x d t .
\end{aligned}
$$

Let $L=L(r)=\mu(r) r^{-1}+\Lambda$ and $\beta \geq 2$. We would like to multiply both sides of inequality (56) by $k^{-2 \gamma-\beta-1}$ and integrate with respect to $k$ from 0 to $\infty$. However, 
$k$ is restricted by $0 \leq k \leq L$. We thus proceed in the following way. Note that

$$
\begin{aligned}
-\int_{0}^{\infty} & \iint_{\{\bar{v}<k\}} \eta^{2} \bar{v}_{T}^{2 \gamma}(k-\bar{v}) \bar{v}_{t} k^{-2 \gamma-\beta-1} d k \\
& +\frac{1}{3} \int_{0}^{\infty} \iint_{\{\bar{v}<k\}} \eta^{2} \bar{v}_{T}^{2 \gamma}|\nabla \bar{v}|^{2} k^{-2 \gamma-\beta-1} d k \\
= & -\int_{0}^{L} \iint_{\{\bar{v}<k\}} \eta^{2} \bar{v}_{T}^{2 \gamma}(k-\bar{v}) \bar{v}_{t} k^{-2 \gamma-\beta-1} d k \\
& +\frac{1}{3} \int_{0}^{L} \iint_{\{\bar{v}<k\}} \eta^{2} \bar{v}_{T}^{2 \gamma}|\nabla \bar{v}|^{2} k^{-2 \gamma-\beta-1} d k \\
& -\int_{L}^{\infty} \iint_{\{\bar{v}<k\}} \eta^{2} \bar{v}_{T}^{2 \gamma}(k-\bar{v}) \bar{v}_{t} k^{-2 \gamma-\beta-1} d k \\
& +\frac{1}{3} \int_{L}^{\infty} \iint_{\{\bar{v}<k\}} \eta^{2} \bar{v}_{T}^{2 \gamma}|\nabla \bar{v}|^{2} k^{-2 \gamma-\beta-1} d k \\
= & I_{1}+I_{2}+I_{3}+I_{4} .
\end{aligned}
$$

The last two integrals, $I_{3}$ and $I_{4}$, can easily be evaluated since $\bar{v} \leq L$. Thus,

$$
\begin{aligned}
I_{3}= & -\int_{L}^{\infty} \iint_{\{\bar{v}<k\}} \eta^{2} \bar{v}_{T}^{2 \gamma}(k-\bar{v}) \bar{v}_{t} k^{-2 \gamma-\beta-1} d k \\
= & -\int_{L}^{\infty} \iint_{\{\bar{v}<k\}} \eta^{2} \bar{v}_{T}^{2 \gamma} \bar{v}_{t} k^{-2 \gamma-\beta} d k \\
& +\int_{L}^{\infty} \iint_{\{\bar{v}<k\}} \eta^{2} \bar{v}_{T}^{2 \gamma} \overline{v v}_{t} k^{-2 \gamma-\beta-1} d k \\
= & -\frac{L^{-2 \gamma-\beta+1}}{2 \gamma+\beta-1} \iint_{\{\bar{v}<L\}} \eta^{2} \bar{v}_{T}^{2 \gamma} \bar{v}_{t} d x d t \\
& +\frac{L^{-2 \gamma-\beta}}{2 \gamma+\beta} \iint_{\{\bar{v}<L\}} \eta^{2} \bar{v}_{T}^{2 \gamma} \overline{v v}_{t} d x d t \\
= & -C_{1} L L^{-2 \gamma-\beta} \iint_{\{\bar{v}<L\}} \eta^{2} \bar{v}_{T}^{2 \gamma} \bar{v}_{t} d x d t \\
& +\frac{L L^{-2 \gamma-\beta}}{2 \gamma+\beta} \iint_{\{\bar{v}<L\}} \eta^{2} \bar{v}_{T}^{2 \gamma} \bar{v}_{t} d x d t \\
& +\frac{L^{-2 \gamma-\beta}}{2 \gamma+\beta} \iint_{\{\bar{v}<L\}} \eta^{2} \bar{v}_{T}^{2 \gamma} \bar{v}_{t} d x d t
\end{aligned}
$$

where

$$
C_{1}=\frac{1}{2 \gamma+\beta-1}-\frac{1}{2 \gamma+\beta} .
$$


Hence,

$$
\begin{aligned}
I_{3}+I_{4}= & C_{1} L^{-2 \gamma-\beta} \iint_{\{\bar{v}<L\}} \eta^{2} \bar{v}_{T}^{2 \gamma} \bar{v}_{t} d x d t \\
& -\frac{L^{-2 \gamma-\beta}}{2 \gamma+\beta} \iint_{\{\bar{v}<L\}} \eta^{2} \bar{v}_{T}^{2 \gamma}(L-\bar{v}) \bar{v}_{t} d x d t \\
& +\frac{L^{-2 \gamma-\beta}}{2 \gamma+\beta} \frac{1}{3} \iint_{\{\bar{v}<L\}} \eta^{2} \bar{v}_{T}^{2 \gamma}|\nabla \bar{v}|^{2} .
\end{aligned}
$$

The integrands in the integrals $I_{1}$ and $I_{2}$ can be estimated by (56) and therefore

$$
\begin{aligned}
I_{1}+I_{2} \leq C \int_{0}^{L} \iint_{\{\bar{v}<k\}}\left[\eta^{2} \bar{v}_{T}^{2 \gamma}+\gamma^{2} \bar{v}_{T}^{2 \gamma-2}|\nabla \bar{v}|^{2} \eta^{2}+\bar{v}_{T}^{2 \gamma}|\nabla \eta|^{2}\right] \\
\cdot\left[(v-k)^{-}\right]^{2} k^{-2 \gamma-\beta-1} d k .
\end{aligned}
$$

Thus, we have so far,

$$
\begin{aligned}
& -\int_{0}^{\infty} \iint_{\{\bar{v}<k\}} \eta^{2} \bar{v}_{T}^{2 \gamma}(k-\bar{v}) \bar{v}_{t} k^{-2 \gamma-\beta-1} d k \\
& \quad+\frac{1}{3} \int_{0}^{\infty} \iint_{\{\bar{v}<k\}} \eta^{2} \bar{v}_{T}^{2 \gamma}|\nabla \bar{v}|^{2} k^{-2 \gamma-\beta-1} d k \\
& \leq \\
& \leq \int_{0}^{L} \iint_{\{\bar{v}<k\}}\left[\eta^{2} \bar{v}_{T}^{2 \gamma}+\gamma^{2} \bar{v}_{T}^{2 \gamma-2}|\nabla \bar{v}|^{2} \eta^{2}+\bar{v}_{T}^{2 \gamma}|\nabla \eta|^{2}\right]\left[(v-k)^{-}\right]^{2} k^{-2 \gamma-\beta-1} d k \\
& \quad-C_{1} L L^{-2 \gamma-\beta} \iint_{\{\bar{v}<L\}} \eta^{2} \bar{v}_{T}^{2 \gamma} \bar{v}_{t} d x d t \\
& \quad-\frac{L^{-2 \gamma-\beta}}{2 \gamma+\beta} \iint_{\{\bar{v}<L\}} \eta^{2} \bar{v}_{T}^{2 \gamma}(L-\bar{v}) \bar{v}_{t} d x d t \\
& \quad+\frac{L^{-2 \gamma-\beta}}{2 \gamma+\beta} \frac{1}{3} \iint_{\{\bar{v}<L\}} \eta^{2} \bar{v}_{T}^{2 \gamma}|\nabla \bar{v}|^{2} d x d t
\end{aligned}
$$

Using again (56), the sum of the last two terms is estimated above by

$$
\begin{gathered}
C \frac{L^{-2 \gamma-\beta}}{2 \gamma+\beta}\left[\iint_{R(1)}\left[\gamma^{2} \bar{v}_{T}^{2 \gamma-2}|\nabla \bar{v}|^{2} \eta^{2}+\bar{v}_{T}^{2 \gamma}|\nabla \eta|^{2}+\eta^{2} \bar{v}_{T}^{2 \gamma}\right]\left[(\bar{v}-L)^{-}\right]^{2}\right] \\
\quad=\int_{L}^{\infty} \iint_{R(1)}\left[\gamma^{2} \bar{v}_{T}^{2 \gamma-2}|\nabla \bar{v}|^{2} \eta^{2}+\bar{v}_{T}^{2 \gamma}|\nabla \eta|^{2}+\eta^{2} \bar{v}_{T}^{2 \gamma}\right]\left[(\bar{v}-L)^{-}\right]^{2} k^{(-2 \gamma-\beta-1)} d k
\end{gathered}
$$


Thus, we have

$$
\begin{aligned}
- & \int_{0}^{\infty} \iint_{\{\bar{v}<k\}} \eta^{2} \bar{v}_{T}^{2 \gamma}(k-\bar{v}) \bar{v}_{t} k^{-2 \gamma-\beta-1} d k \\
& +\frac{1}{3} \int_{0}^{\infty} \iint_{\{\bar{v}<k\}} \eta^{2} \bar{v}_{T}^{2 \gamma}|\nabla \bar{v}|^{2} k^{-2 \gamma-\beta-1} d k \\
\leq & C \int_{0}^{L} \iint_{R(1)}\left[\gamma^{2} \bar{v}_{T}^{2 \gamma-2}|\nabla \bar{v}|^{2} \eta^{2}+\bar{v}_{T}^{2 \gamma}|\nabla \eta|^{2}+\eta^{2} \bar{v}_{T}^{2 \gamma}\right]\left[(v-k)^{-}\right]^{2} k^{(-2 \gamma-\beta-1)} d k \\
& \left.+C \int_{L}^{\infty} \iint_{R(1)}\left[\gamma^{2} \bar{v}_{T}^{2 \gamma-2}|\nabla \bar{v}|^{2} \eta^{2}+\bar{v}_{T}^{2 \gamma}|\nabla \eta|^{2}+\eta^{2} \bar{v}_{T}^{2 \gamma}\right]\left[(v-k)^{-}\right]^{2} k^{(-2 \gamma-\beta-1}\right) d k \\
& -C_{1} L L-2 \gamma-\beta \iint_{\{\bar{v}<L\}} \eta^{2} \bar{v}_{T}^{2 \gamma} \bar{v}_{t} d x d t \\
= & C \int_{0}^{\infty} \iint_{R(1)}\left[\gamma^{2} \bar{v}_{T}^{2 \gamma-2}|\nabla \bar{v}|^{2} \eta^{2}+\bar{v}_{T}^{2 \gamma}|\nabla \eta|^{2}+\eta^{2} \bar{v}_{T}^{2 \gamma}\right]\left[(\bar{v}-k)^{-}\right]^{2} k^{(-2 \gamma-\beta-1)} d k \\
& -C_{1}(2 \gamma+\beta-1) \int_{L}^{\infty} \iint_{R(1)} \eta^{2} \bar{v}_{T}^{2 \gamma} \bar{v}_{t} k^{-2 \gamma-\beta} d k .
\end{aligned}
$$

Now let $T \rightarrow \infty$ to obtain

(57)

$$
\begin{aligned}
& -\int_{0}^{\infty} \iint_{\{\bar{v}<k\}} \eta^{2} \bar{v}^{2 \gamma}(k-\bar{v}) \bar{v}_{t} k^{-2 \gamma-\beta-1} d k \\
& \quad+\frac{1}{3} \int_{0}^{\infty} \iint_{\{\bar{v}<k\}} \eta^{2} \bar{v}^{2 \gamma}|\nabla \bar{v}|^{2} k^{-2 \gamma-\beta-1} d k \\
& \leq C \int_{0}^{\infty} \iint_{\{\bar{v}<k\}}\left[\gamma^{2} \bar{v}^{2 \gamma-2}|\nabla \bar{v}|^{2} \eta^{2}+\bar{v}^{2 \gamma}|\nabla \eta|^{2}+\eta^{2} \bar{v}^{2 \gamma}\right]\left[(\bar{v}-k)^{-}\right]^{2} k^{(-2 \gamma-\beta-1)} d k \\
& \quad+\frac{2 C_{1}}{(2 \gamma+1)} \iint_{R(1)} \eta\left|\eta_{t}\right| \bar{v}^{2-\beta} d x d t .
\end{aligned}
$$

We now analyze the terms in the above inequality that involve $|\nabla \bar{v}|^{2}$. The term on the left becomes

$$
\frac{1}{3(2 \gamma+\beta)} \iint_{R(1)} \eta^{2}|\nabla \bar{v}|^{2} \bar{v}^{-\beta} d x d t
$$

whereas the term on the right can be estimated above by

$$
\frac{C \gamma^{2}}{2 \gamma+\beta-2} \iint_{R(1)} \eta^{2}|\nabla \bar{v}|^{2} \bar{v}^{-\beta} d x d t .
$$

By taking $\gamma$ sufficiently small, this term can be absorbed by the left side of (57). The term on the left side of (57) involving $\bar{v}_{t}$ is equal to

$$
\begin{gathered}
\frac{C_{1}}{2-\beta} \iint_{R(1)} \eta^{2}\left(\bar{v}^{2-\beta}\right)_{t} d x d t, \quad \text { if } \beta>2, \\
C_{1} \iint_{R(1)} \eta^{2}(\log \bar{v})_{t} d x d t, \quad \text { if } \beta=2 .
\end{gathered}
$$


. hus, (57) now yields

$$
\begin{aligned}
& K_{1} \iint_{R(1)} \eta^{2}(f(\bar{v}))_{t} d x d t+\frac{C}{2 \gamma+\beta} \iint_{R(1)} \eta^{2} \bar{v}^{-\beta}|\nabla \bar{v}|^{2} d x d t \\
& \quad \leq C_{1} C \iint_{R(1)}\left(\eta^{2}+|\nabla \eta|^{2}\right) \bar{v}^{2-\beta} d x d t+\frac{2 C_{1}}{2 \gamma+1} \iint_{R(1)} \eta\left|\eta_{t}\right| \bar{v}^{2-\beta} d x d t
\end{aligned}
$$

where $f(\bar{v})=\bar{v}^{(2-\beta) / 2}$ if $\beta>2, f(\bar{v})=\log \bar{v}$ if $\beta=2, K_{1}=C_{1} /(2-\beta)$ if $\beta>2$, and $K_{1}=C_{1}$ if $\beta=2$. Simple estimates of the constants involved and an argument similar to that used in Theorem 3.1 reveal that (58) can be written as

$$
\begin{gathered}
\sup _{t \in\left(t_{1}, t_{2}\right)} \int \eta^{2} f(\bar{v}(x, t))^{2} d x+\int_{t_{1}}^{t_{2}} \int|\nabla(\eta f(\bar{v}))|^{2} d x d t \\
\leq C|\beta| \int_{t_{1}}^{t_{2}} \int\left(\eta^{2}+|\nabla \eta|^{2}+2 \eta\left|\eta_{t}\right|\right) f(\bar{v})^{2} d x d t
\end{gathered}
$$

when $\beta>2$ and as

$$
\begin{aligned}
\int_{t_{1}}^{t_{2}} \int \eta^{2} f(\bar{v})_{t} d x+K \int_{t_{1}}^{t_{2}} \int \eta^{2}|\nabla f(\bar{v})|^{2} d x d t \\
\leq C \int_{t_{1}}^{t_{2}} \int \eta^{2}+|\nabla \eta|^{2} d x d t
\end{aligned}
$$

when $\beta=2 . \quad K$ and $C$ are constants that depend only on the structure (1). These inequalities are precisely of the form needed to implement the Moser iteration scheme which yields weak Harnack inequalities, cf. [AS,T2]. Thus, we have

$$
\underset{R(1 / 2)}{\operatorname{ess} \inf } \bar{v} \geq C \int_{R^{*}(1)} \bar{v} d x d t .
$$

By rescaling, we have that

$$
\begin{aligned}
\bar{u}(r)-\bar{u}(r / 2) & \geq[\bar{u}(r)-\bar{\psi}(r)]-[\bar{u}(r / 2)-\bar{\psi}(r)]^{+} \\
& =\inf _{R(r / 2)} v \geq C^{r} f_{R^{*}(r)}\left[\mu(r)-[u(x, t)-\bar{\psi}(r)]^{+}\right] d x d t-C^{\prime} r,
\end{aligned}
$$

thus establishing the conclusion.

Wc now establish a similar inequality for $[u-\underline{u}(r)]$. We continue to use the assumption and notation introduced in (53) and (54).

4.2 LemMA. There are constants $C$ and $C^{\prime}$ depending only on the structure (1) and $\tau$ in (22) such that

$$
\underline{u}\left(\frac{r}{2}\right)-\underline{u}(r) \geq C f_{R^{*}(r)}[u(x, t)-\underline{u}(r)] d x d t-C^{\prime} r
$$

for all $r$ such that $R(r) \subset \Omega$.

PrOOF. The proof is almost identical to Lemma 4.1, but less complicated. Let $v(z)=[u(z)-\underline{u}(r)]$ and for each positive number $k$, let $w=(v-k)^{-}$. The test function this time is defined as $\varphi=\eta^{2} w$. Since $\varphi \geq 0$, it is admissible in (16) and the proof now proceeds as in Lemma 4.1 by multiplying both sides of (56) 
by $k^{2 \gamma-\beta-1}$. Since there is no restriction on $k$, one may integrate the resulting inequality from 0 to $\infty$ to obtain the desired result.

4.3 REMARK. Observe that Lemmas 4.1 and 4.2 remain valid if the notion of solution to the obstacle problem as discussed in (18) had been adopted. In the proof of Lemma 4.1, the test function $\varphi=-\eta^{2}(v-k)^{-}$was employed. However, it can easily be seen that $(v-k)^{-}=[u-(\mu-k+\bar{\psi}(r))]^{+}$. This is clear if $\mu=k$. If $\mu<k$, note that $v \leq k$ if $(u-\bar{\psi}(r)) \chi_{\{u \geq \bar{\psi}(r)\}} \geq \mu-k$. Thus,

$$
\begin{aligned}
(v-k)^{-} & =(k-v) \chi_{\{v \leq k\}}=\left(k-\mu+(u-\bar{\psi}(r)) \chi_{\{u \geq \bar{\psi}(r)\}}\right) \chi_{\{v \leq k\}} \\
& =\left(k-\mu+\left(u-\bar{\psi}(r) \chi_{\{u \geq \bar{\psi}(r)\}}\right) \chi_{\{u \geq \mu-k+\bar{\psi}(r)\}}\right. \\
& =(k-\mu) \chi_{\{u \geq \mu-k+\bar{\psi}(r)\}}+(u-\bar{\psi}(r)) \chi_{\{u \geq \mu-k+\bar{\psi}(r)\}} \\
& =[u-(\mu-k+\bar{\psi}(r))]^{+} .
\end{aligned}
$$

Hence, we could have used the test function $\varphi=\eta^{2}\left[u-(\mu-k+\bar{\psi}(r)]^{+}\right.$as allowed in $(18)$ to arrive at $(28)$ with $(v-k)^{+}$replaced by $(v-k)^{-}$. Similar considerations show that Lemma 4.2 could be achieved with this alternate notion of solution.

We now establish an estimate for the modulus $\underline{\omega}(r)=u\left(z_{0}\right)-\underline{u}(r)$.

4.4 THEOREM. Let $u \in W^{1,2}(\Omega)$ be a solution as in (15) and (16). Let $A \subset \Omega$ be a set that is not thin at $z_{0}$ and such that

$$
\lim _{\substack{z \rightarrow z_{0} \\ z \in A}} \psi(z)=\bar{\psi}\left(z_{0}\right)
$$

Let

$$
\underline{\psi}(r, A)=\operatorname{ess} \inf \left\{\psi(z): z \in R^{-}(r) \cap A\right\} \quad \text { and } \quad \varepsilon(r)=\bar{\psi}\left(z_{0}\right)-\underline{\psi}(r, A)+r
$$

where the ess sup is taken in the sense of capacity. Then there exist constants $C_{1}$ and $C_{2}$ such that for sufficiently small $s>0$,

$$
\underline{\omega}(r) \leq \varepsilon(r)+C_{1} \exp \left(-C_{2} \int_{2 r}^{s} \operatorname{cap}_{T}\left(\frac{R^{-}(\rho) \cap A}{R^{-}(\rho)}\right) \frac{d \rho}{\rho}\right)
$$

whenever $r \leq s / 2$.

PROOF. Notice that $\operatorname{cap}_{T}\left[R^{-}(r) \cap A\right] \leq \operatorname{cap}_{T}\left[A\left(z_{0}, \varepsilon(r)\right]\right.$ where $A\left(z_{0}, \varepsilon(r)\right)=$ $R^{-}(r) \cap\left\{z: u(z)>u\left(z_{0}\right)-\varepsilon(r)\right\}$. We proceed as in the proof of Theorem 3.3, the only difference being that this time $u$ is a supersolution. Thus, let $k(r)=$ $\bar{\psi}\left(z_{0}\right)-\varepsilon(r)$ and define $w=(u-k(r))^{-}$. Therefore $\nabla u=-\nabla w \chi_{\{u<k(r)\}}$. Let $m(r)=\sup _{R(r)} w$ and define $f(w)=m(r)-w^{2}$. Note that $f=m(r)$ on $A\left(z_{0}, \varepsilon(r)\right)$. As in the proof of Theorem 3.3, define a test $\varphi=\eta \zeta f^{\prime}(w)$ and proceed to obtain an estimate similar to (44):

$$
\begin{aligned}
& \frac{m(r)}{2} \operatorname{cap}_{T}\left(R^{-}(r) \cap A\right) \leq \frac{m(r)}{2} \operatorname{cap}_{T}\left(A\left(z_{0}, \varepsilon(r)\right)\right) \\
& \leq C[m(r)-m(r / 2)+a r] r^{n} .
\end{aligned}
$$

Referring to [GZ, Theorem 2.7], we see that (60) implies that there exist constants $C_{1}$ and $C_{2}$ such that for sufficiently small $s>0$,

$$
m(r) \leq C_{1} \exp \left(-C_{2} \int_{2 r}^{s} A(t) \frac{d t}{t}\right)
$$


whenever $r \leq s / 2$. Here

Thus, we obtain

$$
A(t)=C^{-1} \operatorname{cap}_{T}\left(\frac{R^{-}(t) \cap A}{R^{-}(t)}\right)
$$

$$
u\left(z_{0}\right)-\underline{u}(r)=\bar{\psi}\left(z_{0}\right)-\underline{u}(r)=m(r)+\varepsilon(r),
$$

and the conclusion is established.

We now are able to establish a bound for the modulus $\bar{\omega}(r)=\bar{u}(r)-u\left(z_{0}\right)$. In the following it is assumed that (53) is in force.

4.5 THEOREM. Let $r_{0}=\sup \left\{r: R\left(r, z_{0}\right)=R(r) \subset \Omega\right\}$. Then for $r<\frac{1}{3} r_{0}$ and $\alpha<1$, there are positive constants $C_{1}, C_{2}$, depending only on the structure (1) and $\tau$ in (22) and $\delta>0$ which depends on $\alpha$ as well such that,

$$
\begin{aligned}
\bar{\omega}(r) \leq C_{1} & {\left[\left(r / r_{0}\right)^{\delta} \bar{\omega}\left(r_{0}\right)+\bar{\psi}(\rho)-\bar{\psi}\left(z_{0}\right)\right.} \\
& \left.+\exp \left(-C_{2} \int_{2 \rho}^{s} \operatorname{cap}_{T}\left(\frac{R^{-}(t) \cap A}{R^{-}(t)}\right) \frac{d t}{t}\right)+\rho\right]
\end{aligned}
$$

whenever $s>0$ is sufficiently small and $\rho \leq s / 2$. Here, $\rho=\rho\left(r, r_{0}\right)=r^{\alpha} r_{0}^{1-\alpha}$ and $A$ is as in Theorem 4.4 .

Proof. Appealing to Lemma 4.1, we have

$$
\bar{u}(r)-\bar{u}\left(\frac{r}{2}\right) \geq C f_{R^{*}(r)} \mu(r) d x d t-f_{R^{*}(r)}[u(x, t)-\bar{\psi}(r)]^{+} d x d t-C^{\prime} r .
$$

This may be written as

$$
\begin{aligned}
{\left[\bar{u}(r)-u\left(z_{0}\right)\right]-\left[\bar{u}(r / 2)-u\left(z_{0}\right)\right] \geq } & C\left[\left(\bar{u}(r)-u\left(z_{0}\right)\right)-\left(\bar{\psi}(r)-\bar{\psi}\left(z_{0}\right)\right)\right] \\
& -f_{R^{*}(r)}[u(x, t)-\bar{\psi}(r)]^{+} d x d t-C^{\prime} r
\end{aligned}
$$

or as

$$
\begin{aligned}
\bar{\omega}(r / 2) \leq & (1-C) \bar{\omega}(r)+C^{\prime \prime}\left[\bar{\psi}(r)-\bar{\psi}\left(z_{0}\right)+r\right] \\
& +f_{R^{*}(r)}[u(x, t)-\bar{\psi}(r)]^{+} d x d t .
\end{aligned}
$$

Observe that $\underline{u}(r) \leq \bar{\psi}(r)$ for otherwise $u\left(z_{0}\right) \geq \underline{u}(r)>\bar{\psi}(r) \geq \bar{\psi}\left(z_{0}\right)=u\left(z_{0}\right)$. Hence, by Lemma 4.2 ,

$$
\begin{aligned}
f_{R^{*}(r)}[u(x, t)-\bar{\psi}(r)]^{+} d x d t & \leq f_{R^{*}(r)}[u(x, t)-\underline{u}(r)] d x d t \\
& \leq C[\underline{u}(r / 2)-\underline{u}(r)+r] \\
& =C\left[\underline{u}(r / 2)-u\left(z_{0}\right)+u\left(z_{0}\right)-\underline{u}(r)+r\right] \\
& \leq C \underline{\omega}(r)+C^{\prime} r .
\end{aligned}
$$

Thus,

$$
\bar{\omega}(r / 2) \leq(1-C) \bar{\omega}(r)+C^{\prime \prime}\left[\bar{\psi}(r)-\bar{\psi}\left(z_{0}\right)+r\right]+C \underline{\omega}(r)+C^{\prime} r .
$$

The result now follows from Theorem 4.4 and [T2, Lemma 2.2].

By combining the results of Theorems 4.4 and 4.5, we obtain the following bound on the oscillation $\omega(r)=\bar{u}(r)-\underline{u}(r)$. With the same hypotheses as Theorem 4.5, we have the following corollary. 
4.6 COROLlary. For $r<\frac{1}{3} r_{0}$ and $\alpha<1$, there are positive constants $C_{1}$ and $C_{2}$, depending only on the structure (1) and $\tau$ in (22) and $\delta>0$ which depends on $\alpha$ as well such that,

$$
\begin{aligned}
\omega(r) \leq C_{1}\left[\left(r / r_{0}\right)^{\delta} \omega\left(r_{0}\right)+(\bar{\psi}(\rho)-\underline{\psi}(\rho, A))\right. \\
\left.\quad+\exp \left(-C_{2} \int_{2 \rho}^{s} \operatorname{cap}_{T}\left(\frac{R^{-}(t) \cap A}{R^{-}(t)}\right) \frac{d t}{t}\right)+\rho\right]
\end{aligned}
$$

whenever $s>0$ is sufficiently small and $\rho \leq s / 2$. Here, $\rho=\rho\left(r, r_{0}\right)=r^{\alpha} r_{0}^{1-\alpha}$ and $A$ is as in Theorem 4.4.

In particular, if $\bar{\psi}(r)-\underline{\psi}(r) \leq C r^{\beta}$ for some $\beta>0$, then $A$ can be taken as $R^{n+1}$ and therefore there exists $\delta>0$ such that $\omega(r) \leq C r^{\delta}$.

\section{REFERENCES}

[AS] D. G. Aronson and J. Serrin, Local behavior of solutions of quasilinear parabolic equations, Arch. Rational Mech. Anal. 25 (1967), 81-83.

[BI] Marco Biroli, Nonlinear parabolic variational inequalities, Comment Math. Univ. Carolin. 26 (1985), 23-39.

[BM] M. Biroli and U. Mosco, Wiener estimates for parabolic obstacle problems, Nonlinear Analysis, TMA (to appear).

[CK] L. A. Caffarelli and D. Kinderlehrer, Potential methods in variational inequalities, J. Analyse Math. 37 (1980), 285-295.

[DV] H. Beirao da Veiga, Sulla holderianita delle soluzioni di alcune disequazioni variazionali con condizioni unilatere al bordo, Ann. Mat. Pura Appl. 83 (1969), 73-112.

[FM] J. Frehse and U. Mosco, Irregular obstacles and quasi-variational inequalities of the stochastic impluse control, Ann. Scuola Norm. Sup. Pisa Cl. Sci. (4) 1 (1982), 105-157.

[GZ] R. Gariepy and W. P. Ziemer, A regularity condition at the boundary for solutions of quasilinear elliptic equations, Arch. Rational Mech. Anal. 67 (1977), 25-39.

[GZ2] — , Thermal capacity and boundary regularity, J. Differential Equations 45 (1985), 374388 .

[JMZ] B. Jessen, J. Marcinkiewicz and A. Zygmund, Note on the differentiability of multiple integrals, Fund. Math. 25 (1934), 217-234.

[LSU] O. A. Ladyzhenskaya, V. A. Solonnikov and N. Uraltseva, Linear and quasilinear equations of parabolic type, Transl. Math. Mono., vol. 23, Amer. Math. Soc., Providence, R. I., 1968.

[MO] U. Mosco, Wiener criterion and potential estimates for the obstacle problem, preprint, 1985.

[MP] F. Mignot and J. P. Puel, Inequations paraboliques avec convexe dependant du temps: application aux inequations quasi-variationnelles d'evolution, Arch. Rational Mech. Anal. 64 (1977), 59-91.

[MZ] J. H. Michael and W. P. Ziemer, Interior regularity for solutions to obstacle problems, Nonlinear Analysis, TMA 10 (1987), 1427-1448.

[P] M. Pierre, Problemes d' evolution avec contraintes unilaterales et potentiels paraboliques, Comm. Partial Differential Equations 4 (1979), 1149-1197.

[SV] M. Struwe, and M. Vivaldi, On the Hölder continuity of bounded weak solutions of quasi-linear parabolic inequalities, Ann. Mat. Pura Appl. 139 (1985), 175-189.

[T1] N. S. Trudinger, On Harnack type inequalities and their application to quasilinear equations, Comm. Pure Appl. Math. 20 (1967), 721747.

[T2] _ Pointwise estimates and quasilinear parabolic equations, Comm. Pure Appl. Math. 21 (1968), 205-266.

[Z] W. P. Ziemer, Behavior at the boundary of solutions of quasilinear parabolic equations, J. Differential Equations 35 (1980), 291-305. 